\title{
Supervision and Motion Planning for a Mobile Manipulator Interacting with Humans
}

\author{
E. Akin Sisbot, Aurélie Clodic, Rachid Alami, Maxime Ransan \\ LAAS/CNRS and University of Toulouse \\ 7, Avenue du Colonel Roche \\ 31077, Toulouse, France \\ lastname@laas.fr
}

\begin{abstract}
Human Robot collaborative task achievement requires adapted tools and algorithms for both decision making and motion computation. The human presence as well as its behavior must be considered and actively monitored at the decisional level for the robot to produce synchronized and adapted behavior. Additionally, having a human within the robot range of action introduces security constraints as well as comfort considerations which must be taken into account at the motion planning and control level. This paper presents a robotic architecture adapted to human robot interaction and focuses on two tools: a human aware manipulation planner and a supervision system dedicated to collaborative task achievement.
\end{abstract}

\section{Categories and Subject Descriptors}

I.2.9 [Computing Methodologies]: Artificial IntelligenceRobotics

\section{General Terms}

Design, Experimentation

\section{INTRODUCTION}

Human robot interaction (HRI) brings new challenges for all aspects of robotics. When interacting with humans, robots must adopt an adaptive and legible behavior in order to comply with social rules; such social behaviors are even more crucial when robots are cooperatively accomplishing tasks with a human partner.

Let's consider a simple scenario where a person needs water and asks the robot to bring it. Even this simple scenario hides many tasks, actions and challenges: in how many actions this tasks should be accomplished?, how to grasp a bottle which should be safe and comfortable to grasp for human and for robot?, which path will the robot take to reach to the bottle and to the human by respecting human's comfort?, how the robot should know if the person still needs water

Permission to make digital or hard copies of all or part of this work for personal or classroom use is granted without fee provided that copies are not made or distributed for profit or commercial advantage and that copies bear this notice and the full citation on the first page. To copy otherwise, to republish, to post on servers or to redistribute to lists, requires prior specific permission and/or a fee.

HRI'08, March 12-15, 2008, Amsterdam, The Netherlands.

Copyright 2008 ACM 978-1-60558-017-3/08/03 ...\$5.00. and what to do if he doesn't need it anymore, how the robot will handle the object, what posture and motion should the robot take in order to be understandable and comfortable?, socially acceptable and safe to its human partner? Even if some of these questions can be dealt with separately, one fundamental issue is common to all of them.

Autonomous robots perceive their world, make decisions on their own and perform coordinated actions to carry out their tasks. When interacting with humans, robots need to go beyond these capacities with communication and collaboration abilities.

Several collaboration theories $[9,14,8]$ emphasize that a collaborative task has specific requirements compared to individual ones e.g. since the robot and the person share a common goal, they have to agree on the manner to realize it, they must show their commitment to the goal during execution, etc.

Robotics system have already been built upon these theories $[21,23,25,7]$ and they all show benefits taking these specificities into account. Those system show the need to manage turn-taking between communication partners but also show how it is difficult to interleave task realisation and communication. They also show the need to manage beliefs to maintain common ground in order to make them understandable and predictable to each other, that is generally done via the use of agent modelling. However, it is often the case that it is a human operator that pilots its personal software agent in the system, in our system we consider that all knowledge is on-board the robot and only itself can update it through perception abilities. Finally, today only few systems $[13,7]$ take human into account at all levels of the system but as we said previously its our conviction that it is mandatory.

To obtain a safe and friendly interaction, robot motions also should be generated by taking into account human safety, comfort and preferences. This requires explicit reasoning on humans in motion planning and control level.

In this paper we present the integration of Human Aware Manipulation Planner, a motion planner that reasons on human's kinematic structure, his vision field and preferences and SHARY, a supervision system in an overall architecture dedicated to Human-Robot Interaction.

The paper is organized as follows: section 2 described the general architecture adapted to Human-Robot Interaction, section 3 illustrates a human-aware manipulation planner. A supervision system dedicated to collaborative task achievement is introduced in section 4. Finally an illustrative example is shown in section 5 


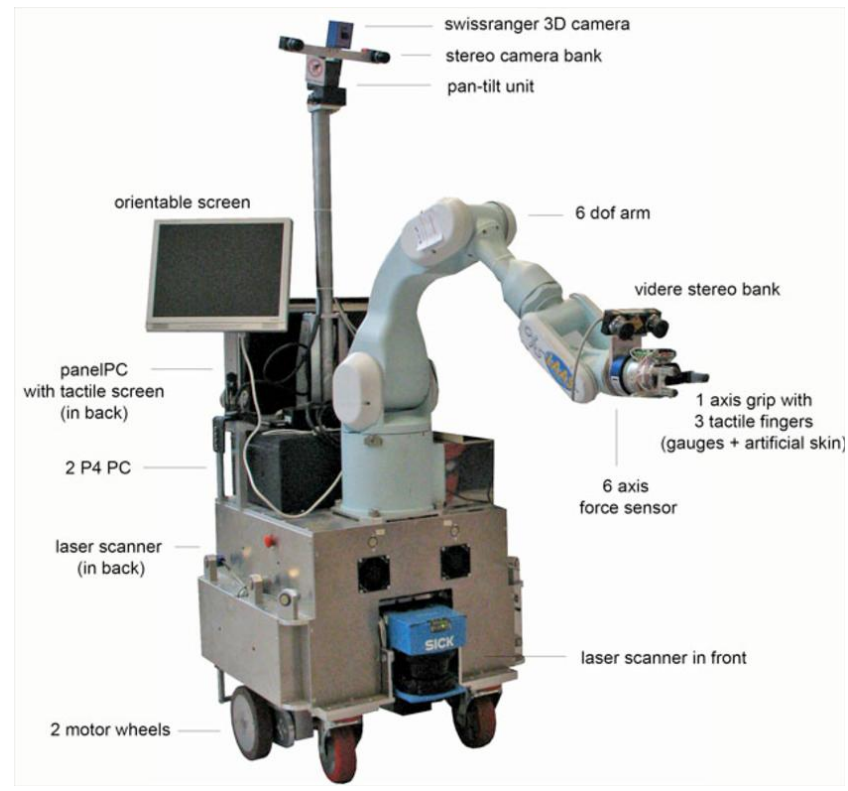

Figure 1: Jido - a mobile manipulator robot

\section{GENERAL ARCHITECTURE}

The goal of the Jido robotic platform (figure 1) is to demonstrate the use and the benefit of a robot in our daily life. For the robot to acquire human intentions, dedicated perception capacities are being developed. Vision algorithms enable the robot to detect, track and identify human faces in its vicinity while a laser based detection system computes their position and orientation. Dedicated motion and manipulation planning algorithms taking into account the presence of humans allow the robot to adopt safe and socially acceptable movements.

We define an overall architecture presented in figure 2 adapted to our context where, human and robot share a common space, potentially a common goal and exchange information through various modalities. An Agenda maintains an overall view of on-going, suspended and future toplevel tasks and ensures consistency between them. A facts database with :

- robot's world knowledge containing a priori data (maps, furniture, objects,...) and contextual data (objects position and owners, environment state,...)

- agent's (InterActionAgent (IAA)) database

and updating management abilities able to interpret perceptual information. Resources are made available to the system: planning resources [3], e.g. Navigation and Manipulation in Human Presence Planner - NHP and MHP [24] but also Human Aware Task Planner - HATP [19] and communication schemes to manage interaction within a task. Using these components, Shary ensures multi-level collaborative tasks achievement dealing with tasks execution and communication that supports it.

In the sequel, we present two components of this architecture : Manipulation in Human Presence, an execution and supervision system called SHARY and a first instanciation of this architecture using these two components on a real system.

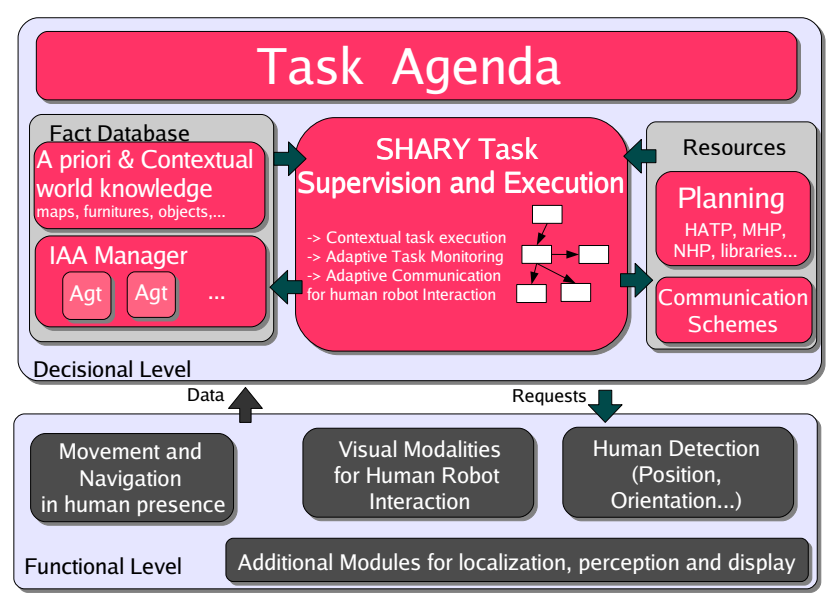

Figure 2: An architecture dedicated to HumanRobot interaction : An Agenda maintains an overall view of on-going, suspended and future tasks,fact database include robot's world knowledge and robot's knowledge concerning other agents, Shary ensure multi-level collaborative tasks achievement with the help of resources: planning system and communication scheme. The functional level is composed of many modules interacting with decisional and hardware level. These modules processes the data acquired from robot sensors and accomplish tasks given by decisional level.

\section{MHP - MANIPULATION IN HUMAN PRESENCE}

This module is an implementation of the Human Aware Manipulation Planner[24]. When humans and robots cohabit in the same environment and accomplish tasks together, the notion of human safety becomes the biggest concern. In industrial environments, safety concerns are reduced to minimum by isolating robots from humans. Eventhough absence of humans in robot's working environment solves safety problems, this isolation implies an absence of interaction between robots and humans which is the major interest of HRI field. As we cannot isolate the robot, the notion of safety becomes very critical and should be studied in every aspects[1].

To achieve safe and friendly motions, several approaches, e.g. danger index minimization[18], symbolic object handling planning[12], have been proposed. We believe that a motion planner reasoning explicitly on humans and placing itself between the symbolic and control levels will benefit highly the quality, the safety and the friendliness of the interaction.

User studies with humans and robots [17][11][22] provide a number of properties and non written rules/protocols [15] of human-robot or human-human interactions.

The Human Aware Manipulation Planner, which is based on these user studies, produces not only physically safe paths but also comfortable, legible, mentally safe paths[20]. Our approach is based on separating the whole problem of manipulation, e.g a robot giving an object to the human, into 3 stages: 
- Finding spatial coordinates of the point where the object will be handled to the human,

- Calculating the path that the object will follow from its resting position to the human hand as it was a free flying object,

- Adapting the whole body motion of the robot along with its posture for manipulation.

All these items must be calculated by taking explicitly into account the human partner to maintain his safety and his comfort. Not only the kinematic structure of the human, but also his vision field, his accessibility, his preferences and his state must be reasoned in the planning loop in order to have a safe and comfortable interaction.

\subsection{Finding Object Handling Position}

Before handling an object to a person, the robot should know the place where the exchange will take place. As the robot is placed in a close interaction scenario with a person, classical motion planners where only the feasibility and the safety of the motion are guaranteed, can end up with very uncomfortable yet feasible paths. Also because of the unpredictability of human's motions, a safe path can be harmful if it does not fullfill the legibility requirement.

We use 3 properties, called "safety", "visibility" and "arm comfort", to find a comfortable object exchange position. Each property is represented by a cost function $f\left(x, y, z, C_{H}, \operatorname{Pre} f_{H}\right.$ for spatial coordinates $(x, y, z)$, a given human configuration $C_{H}$ and his preferences Pref $f_{H}$ when handling an object (e.g left/right handiness, sitting/standing, etc.). This function calculates the cost of a given point around the human by taking into account his preferences, his accessibility his vision field and his state.

- Safety : From a pure safety point of view, the farther the robot places itself, the safer the interaction is. The safety cost function $f_{\text {safety }}$ is a decreasing function according to the distance between the human $H$ and object coordinates $(x, y, z)$. The Pref $f_{H}$ affects the function result according to human states, e.g. sitting, standing, and preferences. The cost of each $(x, y, z)$ around the human is inversely proportional to the distance to the human and illustrated in Figure 3-a.

- Visibility : To make the robot motion legible, a first step is to make the handling position as visible as possible. If possible, object handling should occur in a position in human's field of view and a position that requires minimum effort for human to see. We represent this property with a visibility cost function $f_{\text {visibility }}$.

Alone this function represents the effort required by the human head and body to get the object in his field of view. With a given eye motion tolerance, a point $(x, y, z)$ that has a minimum cost is situated in the cone situated directly in front of human's gaze direction (Figure 3-b). For this property, the Pref $f_{H}$ can contain the eye tolerance for human as well as any preferences or disabilities that he can have.

- Arm Comfort : The final property assures the object to be positioned to a place where the human needs to make the minimum effort to reach. This is calculated by a cost function $f_{\text {arm Comfort }}$ which returns costs representing how comfortable for human arm to reach at a given point $(x, y, z)$. In this case $\operatorname{Pref} f_{H}$ value can contain left/right handiness as well as an other preference.

The inverse kinematics of human arm is solved by IKAN[26] algorithm which returns a comfortable configuration among other possible ones because of the redundancy of the arm structure.

The comfort cost of a point, $f_{\text {arm Comfort }}$, is calculated by merging the human arm joint displacement and the arm's potential energy to reach that point (Figure 3-c)

To find the object handling point, we determine the point that minimizes the weighted combination of these 3 properties. The resulting point will be the target point for the robot to carry the object. Figure 3-d shows coordinates of a found point for a bottle that is calculated by taking into account the 3 properties stated above. This point is visible, safe and easily reachable for the human.

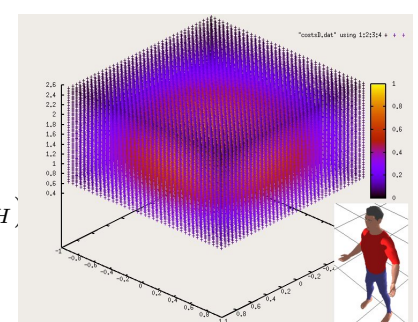

(a)

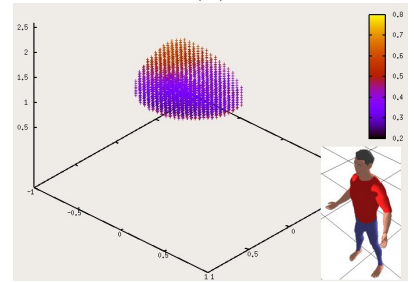

(c)

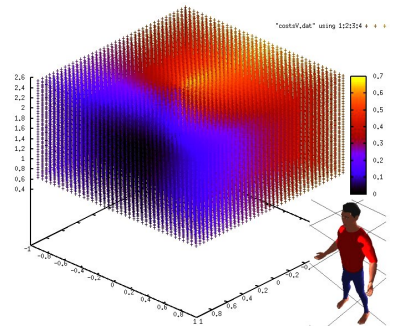

(b)

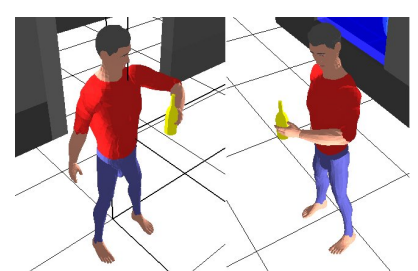

(d)
Figure 3: a- Safety function can be mapped as a protective bubble around the human. b- Visibility function; point that are difficult to see have higher costs. c- Left arm comfort function used with its symmetric right arm function. d- The resulting handling position point is comfortable and safe.

\subsection{Finding Object Path}

Although the object handling point is safe and comfortable, the motion of the robot to reach this point should also be human friendly. Thus reasoning on human's comfort is necessary to find the path that the object will follow.

To find this path we use a 3D grid based approach which we build around the human. This grid contains a set of cells with various costs derived from the relative configuration of the human, his state, his capabilities and preferences.

A combination of safety and visibility functions is used for the $f_{\text {path }}$ cell cost function. With the use of these two, a path that minimizes cell costs will be safe and visible to the 
human:

$$
f_{\text {path }}(x, y, z)=\alpha_{1} f_{\text {safety }}(x, y, z)+\alpha_{2} f_{\text {visibility }}(x, y, z)
$$

After the construction of this $3 \mathrm{D}$ grid, an $\mathrm{A}^{*}$ search is conducted from the initial object position to the object handling position that minimizes the sum of $f_{\text {path }}$ of cells all along the path. This path, illustrated in figure 4, will be the path that the object and the robot's hand will follow.

\subsection{Producing Robot Motion}

The third and final stage of planning consists of finding a path for the robot to follow object's motion. Object's motion is computed as it was a freeflying object. But in reality, it is the robot who holds the object and who will make the object follow it's path.

To adapt the robot structure to the object's motion, we use Generalized Inverse Kinematics [27][4] algorithm. This method allows to manage multiple tasks with priorities and to easily integrate the planner to different type of robots.

At the end of this stage we obtain a path for the robot which is safe, visible and comfortable to the human as we took into account his accessibility, field of view and his preferences.

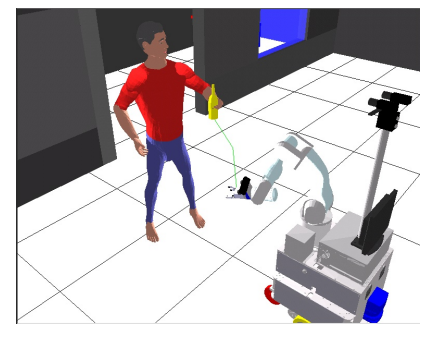

Figure 4: Objects path is calculated as it is a freeflying body. Robot arm is adapted to object motion.

\section{SHARY - HRI SUPERVISION SYSTEM}

\subsection{Collaborative Task Achievement}

When performing collaborative tasks, robots and humans are supposed to share a common goal; therefore not only do they have to agree on the manner to realize it, but they must also show their commitment to the goal during the execution. It is necessary for the human to perceive robot intentions in order to understand its behavior and trust robot decisions. On the other side the robot must monitor human behaviors in order to model their activities and understand their meaning within the current execution context. These observations have raised the need for a new task execution and supervision system; when performing collaborative tasks, robots must not only plan a legible set of actions to achieve a desired goal but they should also actively monitor human commitment. Furthermore robots must close the loop with their human partner by informing them of relevant events (success and failure of actions, plan modification, current intentions).

One of the scenarios that we have been working on consists in a Give Object task where the robot holds out a bottle to a person situated in its vicinity. Indeed this task could be performed in a standard "robocentric" manner where the robot follows predefined steps:- robot handing the bottle in human direction - the person taking the bottle - then Jido retracting its arm. The task will be realized but could it be considered satisfying from a human robot interaction perspective? What happens if the human does not follow exactly the protocol coded in the robot? Who imposes the rhythm of the overall execution ? How does the human know, step by step, about the robot intentions ? Does the robot even have intentions ? In such a case, from a human robot interaction point of view, the task is realized in an open loop where no feedback is given nor perceived by the robot.

A large set of events could occur within this Give Object context implying that the "robocentric" execution stream previously described is only one (out of many) possible way of realizing the task. For instance the person receiving the bottle could turn around, retract his arm, walk away from the robot or even grasp the object during the robot arm movement. Those behaviors should be monitored and analyzed in order to produce meaningful events understandable within a collaborative task achievement.

\subsection{Approach and Concepts}

Joint intention theory [10] states that a joint task cannot be interpreted as a set of individual ones. Whenever agents are performing collaborative tasks they need to share beliefs about the goal they want to achieve. This Joint Persistent Goal (JPG) exists if the agents involved in task the achievement process mutually believe:

- the goal is not yet achieved,

- they want the goal to be achieved,

- until the goal is mutually known to be achieved, unachievable, or no longer relevant, they should persist in holding the goal.

Even though beliefs are the atomic entities for collaborative task achievement we are currently placing ourselves at a higher level of abstraction in which the central notion is the communication act. Communication acts represent the exchange of a piece of information between two agents and can be realized by dialog (oral/visual), by expressive motion or by a combination of the two. It enables each agent to communicate their beliefs about the task to be realized in order to share mutual knowledge and to agree on execution plans and synchronizes their behaviors [5]. Communication acts are defined by a name which characterizes the object of the communication and by a step which defines the evolution of this object during the interaction. We have defined a set of communicative acts that we found mandatory in the framework of task achievement [6]. At any time, both the user and the robot can propose the following task-based communication acts:

- ask: proposing a task,

- propose_plan: proposing a plan (recipe) for a certain task,

- modify_plan: proposing a modification of the current plan for a certain task,

- give_up: abandons a task (e.g., because the task becomes impossible). For the robot this is a way to announce that it is unable to achieve the task. 
- cancel: voluntary cancelation of a task,

- end_task: announces that the task has been done,

- realize: announces that the task performance will start.

For the robot to close the loop with the human we introduce the notion of communication scheme which connects communication acts in a meaningful way. Communication schemes define both the expected incoming communication acts as well as the next communication act to be executed. Figure 5 describes two communication schemes that are currently running. The one on the left is used for joint tasks while the one on the right is used for individual tasks. For instance, when executing the realize act communication act we can see that the robot is expecting: - the human to suspend the task ( $\mathrm{H}$ : suspend act) - the human to give up the task (H:give_up act) - or the human to end the task ( $\mathrm{H}:$ end act).

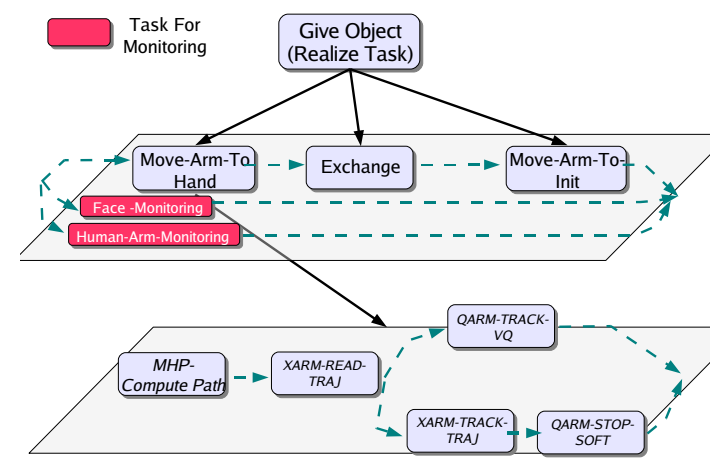

Figure 6: Give Object task decomposition. Tasks written in italic are atomic tasks; they communicate directly with the robot functional layer

When executed, communication acts are decomposed into a partially ordered task tree. This tree contains tasks that effectively perform the communication act but it also defines tasks to monitor human commitment. Figure 6 illustrates the task decompositions for the robot to give an object. The face monitoring makes sure that the human partner is looking at the robot while the arm monitoring checks that the human does not retract his hand while the arm is moving. Those two tasks can generate events such as $\mathrm{H}$ : suspend act which are taken into account by the supervision system. For instance the arm movement can be temporarily interrupted if the person does not face the robot (absence of attention).

\subsection{SHARY Implementation}

As said previously the goal of the SHARY supervision system is to execute tasks in a human robot interaction context. Figure 7 describes the robot general design based on a 3 layer architecture [2]. Human communication acts are perceived via dedicated perception modules: a face detection system is able to identify people recorded in a database, a laser based positioning system can determine human position and orientation as well as basic movement behaviors, finally a touch screen can inform the supervisor about buttons pressed.
The SHARY supervision system consists of two main components: a "task and robot knowledge data base" and a "task refinement and execution engine".

The "task and robot knowledge data base" is composed of:

- Task achievement Communication schemes: store transitions between communication acts as well as expected incoming communications act during execution as shown figure 5; it is fully decoupled from task information.

- Act/Task recipes and monitors library: stores the different recipes (task-tree decomposition) that compute appropriate sub-tasks for both task realization and monitoring.

- Contextual Environment Knowledge: stores information about the current state of the world including internal robot and environment information as well as human modeling data.

Besides the contextual environment information, this robot knowledge is completely independent from the execution context. It contains uninstantiated methods that can be used in different robotic platform with no modifications except for atomic tasks which are usually robot specific. During the execution, this knowledge is used and instantiated in order to produce a context-dependent plan.

The second component of SHARY is "task refinement and execution engine" that embed the decision mechanisms which are responsible for:

- Execution of the communication acts in the current context. It consists in incremental refinement and execution of the current plan.

- Communication management. It monitors human commitment and takes appropriate decisions.

SHARY is implemented using the openPRS [16] procedural reasoning system. Figure 7 gives an example of how the communication library as well as one of the get Recipe functions are encoded. SHARY is currently being used in the Jido robotic platform.

\section{EXAMPLE: BRING-OBJECT COLLABORATIVE TASK}

To illustrate the interest of the human aware motion planner as well as the SHARY supervision system we designed a scenario in which the Jido robotic platform brings an object to a person situated in its vicinity.

The robot starts by a monitoring task which consists in the surveillance of the vicinity until a human stops. $\mathrm{Hu}-$ man detection automatically appends the task BringObject to its current plan and starts the execution. The first communication act that Jido executes is the BringObject: $\mathrm{R}$ :ask act during which its reaches the human and talks to him. ("Do you want the bottle?"). If the human stays in front of the robot, jido interprets this a "YES" i.e. BringObject: $\mathrm{H}$ :ask agree. (Further implementation will integrate speech recognition for better quality human robot interaction). The robot supervisor switches to the BringObject: $\mathrm{R}$ :realize act communication act. This communication act is decomposed into 4 sub-tasks:

- Goto (topological-position BOTTLE-POS) 

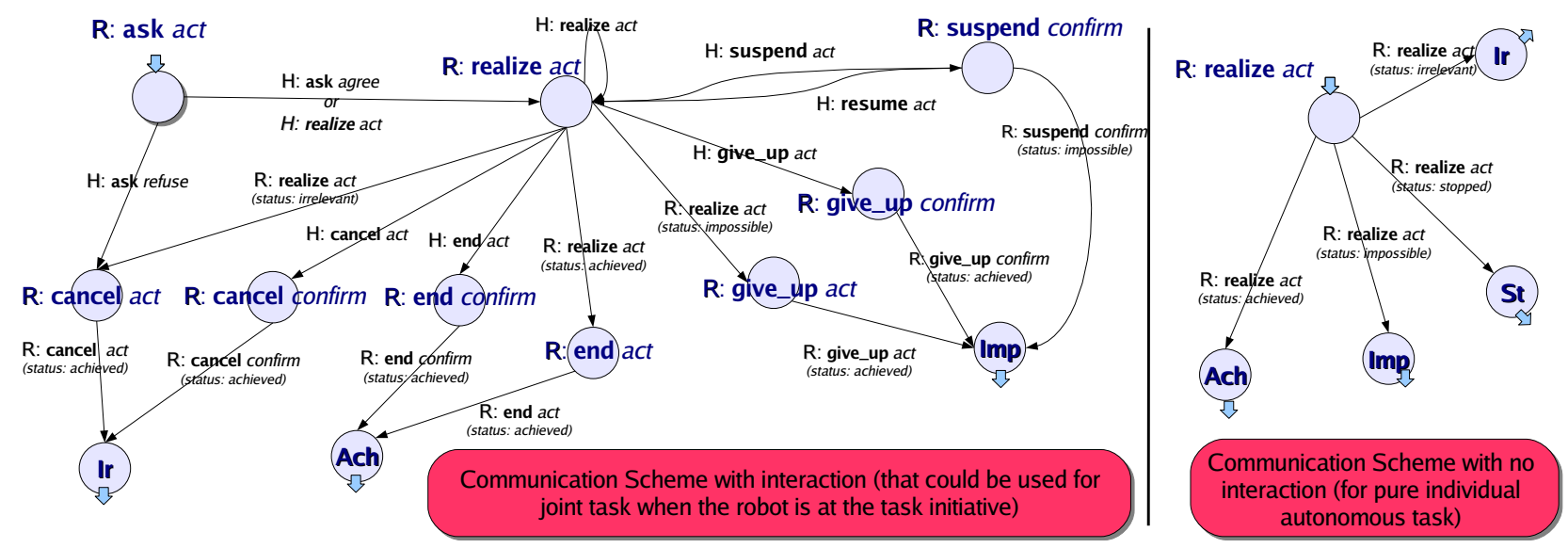

Figure 5: Example of two communication schemes (R:Robot, H:Human realizing the act) currently implemented. The one on the left can typically be used for joint tasks when the robot has the task initiative. The one on the right has no explicit communication, it is used for individual autonomous tasks

- Pick-Up (object BOTTLE)

- Goto (human PERSON-1)

- Give-Object (person PERSON-1) (object BOTTLE)

Only the Give-Object task is joint, the Goto and PickUp task are considered individual.

Jido then goes to the location of the bottle taking into account the human presence. At the time the video was recorded the color based grasping system was not yet implemented therefore the pick-up task was setup so that the arm was moved to a certain configuration and then the robot was waiting at $\max 30$ s for the object to be placed inside the gripper. If the object is detected then the task is considered successfully achieved by the supervision system. In such a case the robots keeps on executing the plan by navigating back to the human and giving him the object. We also tested execution error by intentionally refusing to place the object in the gripper. The pick up task was found to be impossible (Bring-Object R:realize act (status impossible)) to achieve which made SHARY to abandon the task (see the communication on the left of figure 5). Since the BringObject task is collaborative, SHARY executes the BringObject R:give_up act communication act which is decomposed into the following task:

- MoveArm (position INIT-POS)

- Goto (human PERSON-1)

- Speak (sentence "Sorry I could not perform the task")

- Goto (topological-position JIDO-WAITING-ZONE)

Figure 8 shows the two different execution streams of the Bring-Object task.

\section{CONCLUSION}

In this paper, we have presented two key components of the Human Aware Robot Architecture: Human Aware Manipulation Planner that produces paths for robot's arm that fulfills no only safety requirement but also comfort needs, and SHARY, a supervision system adapted for collaborative tasks between humans and robots. More examples and videos can be found at http://www.laas.fr/ easisbot/hri08.

This is a first implementation of a system and a scenario that will be extended toward more complex situations where multiple humans are present and multiple tasks need to be done. Strengthening the link between motion planners and and supervisor with multiple monitor will result a better robot reaction to human states.

In the near future, the supervision system will be improved with the integration of an on-line planning ability that explicitly considers Human-Robot Interaction and Collaborative Problem Solving. Also the manipulation planner will use probabilistic methods to ensure the continuity between base motion and arm motion, thus making a moving and handling motion at the same time. The system will then reach a level where we plan to perform benchmarks and experiment with naive users.

\section{ACKNOWLEDGMENTS}

The work described here was conducted within the EU Integrated Project COGNIRON funded by the E.C. Division FP6-IST under Contract FP6-002020.

\section{REFERENCES}

[1] R. Alami, et al. Safe and dependable physical human-robot interaction in anthropic domains: State of the art and challenges. In IEEE IROS'06 Workshop on pHRI - Physical Human-Robot Interaction in Anthropic Domains, 2006.

[2] R. Alami, R. Chatila, S. Fleury, M. Ghallab, and F. Ingrand. An architecture for autonomy. Internatonal Journal of robotics Research, Special Issue on Integrated Architectures for Robot Control and Programming, 17(4), 1998.

[3] R. Alami, A. Clodic, V. Montreuil, E. A. Sisbot, and C. Raja. Toward human-aware robot task planning. In AAAI Spring Symposium "To boldly go where no human-robot team has gone before", Stanford, USA, March 27-29 2006. 


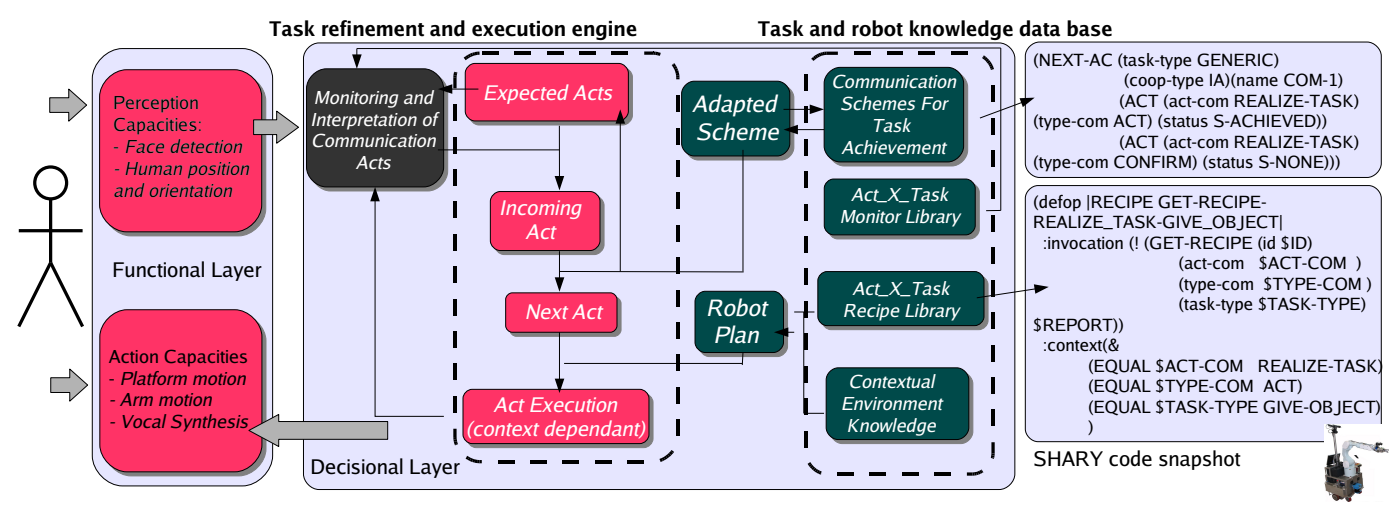

Figure 7: General Description of Shary (at a given task level inside a hierarchy of tasks) : when the task is created, communication scheme associated to the task is instanciated according to the task, the context and concerned agent $=$ Adapted Scheme . This scheme gives the first act to execute. Recipe corresponding to that act (precisely to this act_X_task) is instanciated by the help of a recipes library : Recipe. During Act Execution, communication and execution monitoring is done through wait on Expected Acts. When a monitor is triggered Incoming Act, i.e. when a expected act happend, current act is stopped and the answer is instanciated given the communication scheme Next Act. And so on...

[4] P. Baerlocher and R. Boulic. An inverse kinematics architecture enforcing an arbitrary number of strict priority levels. The Visual Computer, 2004.

[5] C. Aurelie, R. Maxime, A. Rachid, and M. Vincent. A management of mutual belief for human-robot interaction. In In proceedings of IEEE Int. Conf. on Systems, Man and Cybernetics (SMC), 2007.

[6] C. Aurelie, A. Rachid, M. Vincent, L. Shuyin, W. Britta, and S. Agnes. A study of interaction between dialog and decision for human-robot collaborative task achievement. RO-MAN, 2007.

[7] C. Breazeal. Towards sociable robots. Robotics and Autonomous Systems, 42(3):167-175, 2003.

[8] H. H. Clark. Using Language. Cambridge University Press, 1996.

[9] P. R. Cohen and H. J. Levesque. Teamwork. Nous, 25(4):487-512, 1991.

[10] P. R. Cohen, H. J. Levesque, and I. Smith. On team formation. Contemporary Action Theory, 1998.

[11] K. Dautenhahn, et al. How may i serve you?, a robot companion approaching a seated person in a helping context. ACM/IEEE Int. Conference on HRI, Salt Lake City, Utah, USA, 2006.

[12] A. Edsinger and C. Kemp. Human-robot interaction for cooperative manipulation: Handing objects to one another. RO-MAN, 2007.

[13] T. W. Fong, C. Kunz, L. Hiatt, and M. Bugajska. The human-robot interaction operating system. In Human-Robot Interaction Conference. ACM, 2006.

[14] B. J. Grosz and S. Kraus. Collaborative plans for complex group action. Artificial Intelligence, 86:269-358, 1996.

[15] E. T. Hall. The Hidden Dimension. Doubleday, Garden City, N.Y, 1966.

[16] F. Ingrand, R. Chatila, R. Alami, and F. Robert. Prs: A high level supervision and control language for autonomous mobile robots. ICRA, New Orleans, 1996.

[17] K. Koay, E. A. Sisbot, D. A. Syrdal, M. Walters,
K. Dautenhahn, and R. Alami. Exploratory study of a robot approaching a person in the context of handling over an object. AAAI 2007 Spring Symposia, Palo Alto, California, USA, 2007.

[18] D. Kulic and E. Croft. Pre-collision safety strategies for human-robot interaction. Autonomous Robots, 22(2), 2007.

[19] V. Montreuil, A. Clodic, and R. Alami. Planning human centered robot activities. In IEEE Int. Conf. on Systems, Man and Cybernetics (SMC), 2007.

[20] S. Nonaka, K. Inoue, T. Arai, and Y. Mae. Evaluation of human sense of security for coexisting robots using virtual realit, 1st report. In Proc. (IEEE)

International Conference on Robotics $\&$ Automation, New Orleans, USA, 2004.

[21] C. Rich and C. L. Sidner. Collagen: When agents collaborate with people. Proceedings of the first international conference on Autonomous Agents, 1997.

[22] K. Sakata, T. Takubo, K. Inoue, S. Nonaka, Y. Mae, and T. Arai. Psychological evaluation on shape and motions of real humanoid robot. RO-MAN, 2004.

[23] C. L. Sidner, C. Lee, C. Kidd, N. Lesh, and C. Rich. Explorations in engagement for humans and robots. Artificial Intelligence, 166(1-2):140-164, 2005.

[24] E. A. Sisbot, L. F. Marin, and R. Alami. Spatial reasoning for human robot interaction. In IEEE/RSJ IIROS, San Diego, USA, 2007.

[25] M. Tambe. Towards flexible teamwork. Journal of Artificial Intelligence Research, 7:83-124, 1997.

[26] D. Tolani, A. Goswami, and N. Badler. Real-time inverse kinematics techniques for anthropomorphic limbs. Graphical Models and Image Processing, 62:353-388, 2000.

[27] K. Yamane and Y. Nakamura. Natural motion animation through constraining and deconstraining at will. IEEE Transactions on Visualization and Computer Graphics, 9:352 - 360, 2003. 


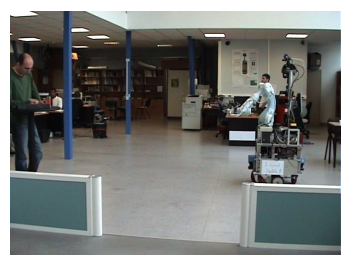

Jido detects a human in its vicinity. A Bring Object interested by a bottle. task is inserted in the The human approves. plan. Jido starts moving to the human thanks to the MHP motion planner.
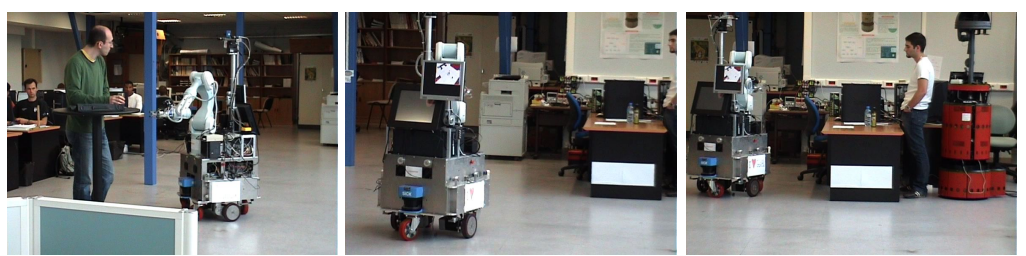

Jido goes to the location of the bottle

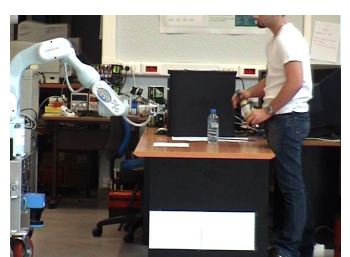

Since autonomous grasping is not yet sufficiently robust, the bottle is placed in the gripper.

\section{Successful Grasping Case}
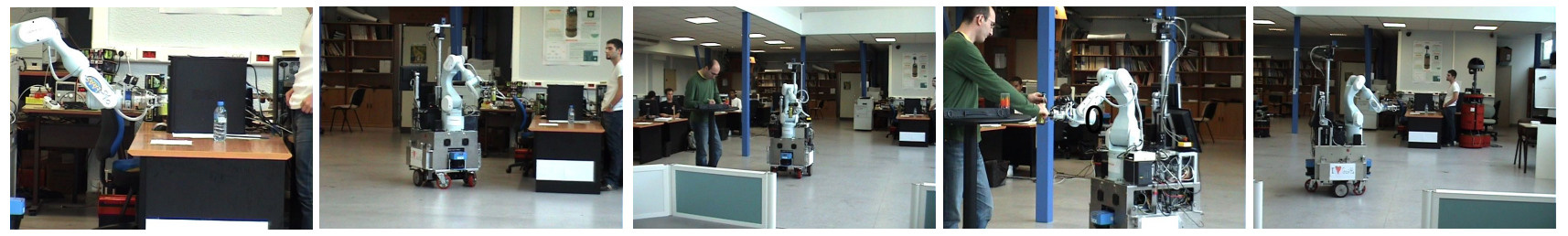

Jido detects that the bottle Jido executes the path computed by the navigation is present and start planner while avoiding obstacles. moving back to the human position.

Jido gives the object to the human by executing a human friendly path computed by MHP. While the arm is moving, the human grasp the bottle, this behavior being interpreted as ( $\mathrm{H}$ :End-Task act) by shary.

Jido returns to the waiting zone.

\section{Unsuccessful Grasping Case}

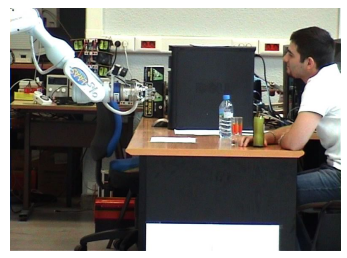

The bottle is not placed in SHARY follows the collaborative communication the gripper, simulating a scheme and execute the Give-Up task. This implies pick-up error.
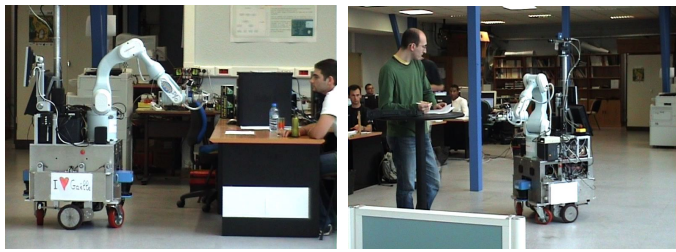

that the robot informs the human partner about the task failure closing, therefore closing the Human-Robot Interaction loop.
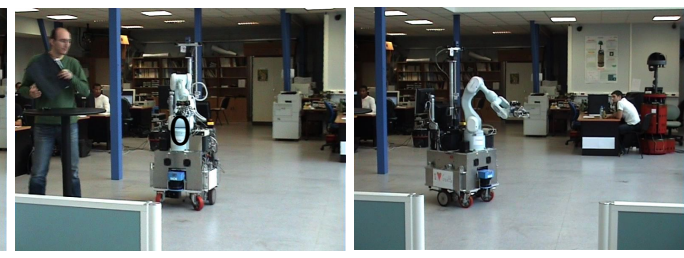

Once Jido has informed the human, it returns back to its waiting zone.

Figure 8: Bring Object task execution 